\title{
Heartbreak: A case of post-infarction cardiogenic shock
}

\author{
Ashes Mukherjee (D) and Jason Fong \\ Department of Emergency Medicine, Armadale Hospital, Armadale, Western Australia, Australia
}

\begin{abstract}
Ventricular septal rupture is a rare but catastrophic complication of acute myocardial infarction. Although it has declined in incidence since the introduction of percutaneous coronary intervention, there hasn't been a significant change of mortality from the condition. In the chain of survival, prompt diagnosis and definitive surgery form the cardinal links. Prolonged medical management is not a feasible option as it is likely to be futile but the aim should be to reduce afterload with the help of intra-aortic balloon pump or support with ventricular assist devices. Echocardiography sits at the heart of the diagnosis of this time critical condition and will guide accurate therapy and intervention. We present the first reported case from an Australian emergency department, where the echocardiography done by the emergency physician clinched the diagnosis. We emphasise here the paramountcy of emergency physicians being proficient in basic echocardiography to achieve rapid diagnosis. Once diagnosed it is critical to have an individual case-tailored multi-disciplinary discussion between emergency medicine, cardiothoracic surgery, cardiology and intensive care as to plan the optimal timing of surgery.
\end{abstract}

Keywords: cardiogenic shock, emergency echo, TTE, ventricular septal rupture.

\section{Introduction}

Ventricular septal rupture (VSR) has a bimodal incidence, the first being within the first $24 \mathrm{~h}$ post-myocardial infarction (MI) and the second being 3-5 days post MI. ${ }^{1}$ Cardiogenic shock from a mechanical complication of acute myocardial infarction (AMI) is one of the most lethal diseases in cardiovascular medicine. Typically, the early mechanical complications of AMI present either as papillary muscle rupture (PMR), ventricular septal rupture $(0.2 \%)$, free wall rupture (FWR), a combination of PMR + VSR or VSR + FWR. ${ }^{2}$

Medical management is not a feasible therapeutic option for most patients, and mainly provides support for surgical intervention, which though challenging and associated with a high mortality, remains the treatment of choice. Prompt diagnosis is the key and echocardiography performed at the earliest will clinch this rare diagnosis. As we demonstrate, an emergency physician's prowess with bedside echocardiography expedites the diagnosis and management of this time critical condition.

\section{Case presentation}

A 55-year-old normally fit and well male with no previous cardiac history, self-presented to our emergency department driven in by his wife with the complaint of chest tightness over the

Correspondence to email ashes.mukherjee@health.wa.gov.au doi: 10.1002/ajum.12123 previous $6 \mathrm{~h}$. There had been a presentation to his general practitioner a week earlier for general lethargy and an episode of diaphoresis which was attributed to viral illness.

He was pale and diaphoretic on presentation but alert and had no increased work of breathing. His initial vitals showed a respiratory rate of 16 , heart rate of $120, \mathrm{BP}-90 / 56$ a capillary refill time of $<2 \mathrm{~s}$ and $\mathrm{SpO}_{2}$ (peripheral oxygen saturation) of $98 \%$ on room air. His chest auscultation did not reveal any added sounds and he had good air entry. Abdominal examination was normal and he had no neurological deficit.

His ECG (Figure 1) revealed ST elevation in leads II, III and aVF along with ST depression in I, aVL, V3-V6 and right bundle branch block, signs of an inferior ST elevation Myocardial infarction (STEMI). The symptoms and ECG findings were thus consistent with STEMI. He was later found to have a white cell count of $10.50 \times 10^{*} \mathrm{~g} / \mathrm{L}$, serum creatinine of $203 \mu \mathrm{mol} / \mathrm{L}$ and cardiac troponin of $8800 \mathrm{ng} / \mathrm{L}$.

Being a hospital with no inpatient cardiology services, the cardiology team at the referral hospital $30 \mathrm{~km}$ away were contacted for urgent PCI. This referral hospital unfortunately does not though have any cardiothoracic surgical services. Following the local AMI protocol for dual antiplatelet therapy, he was given Aspirin \& Ticagrelor along with intravenous heparin.

However, prior to the arrival of the ambulance for the transfer, a point of care echocardiogram was performed by a second 


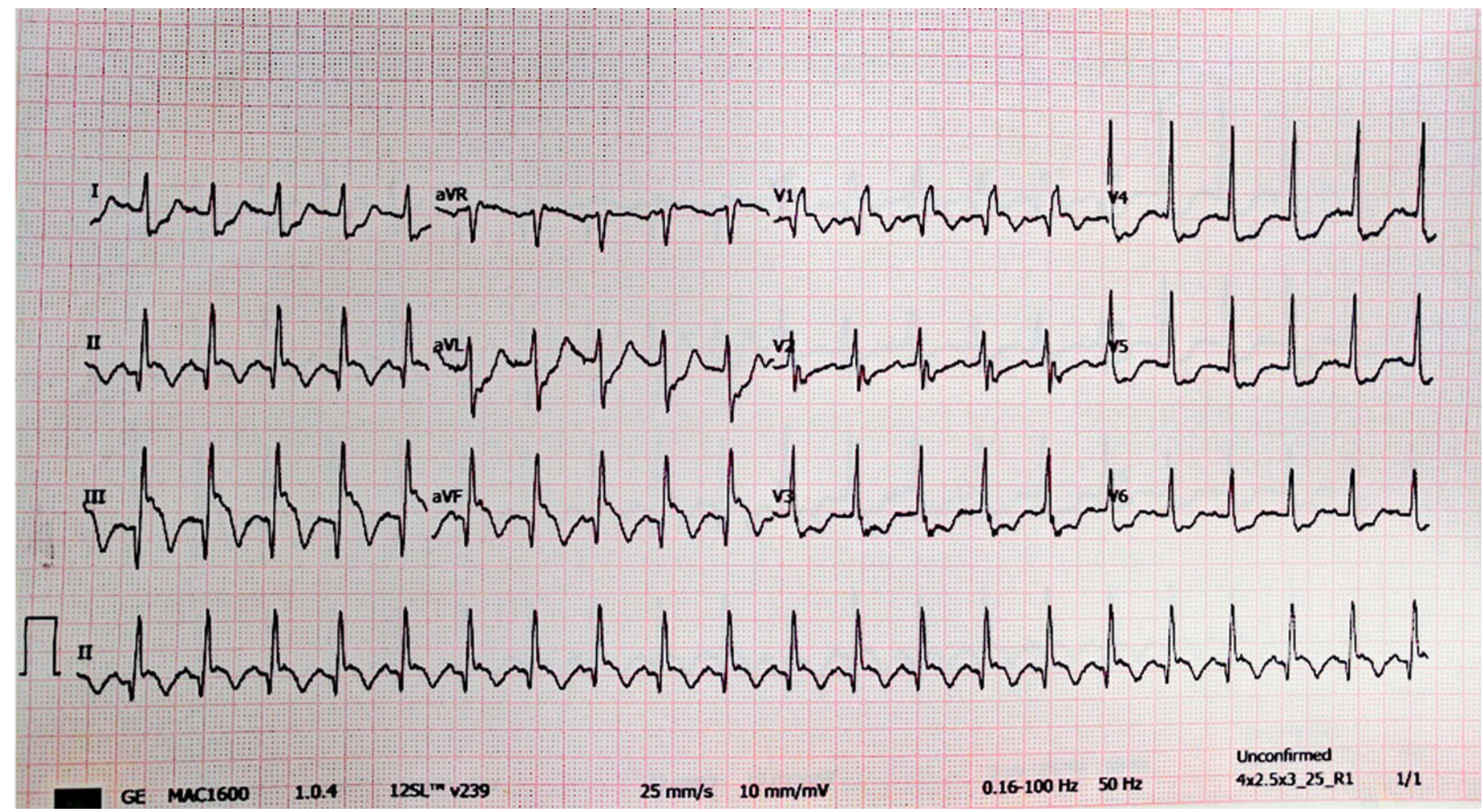

Figure 1: ECG Demonstrating an Inferior ST Elevation Myocardial Infarction with Tachycardia.

emergency physician (EP) because of the concern of early cardiogenic shock. Bedside echocardiography was performed using a Phillips SPARQ portable ultrasound machine with a S4$2 \mathrm{MHz}$ cardiac phased array transducer. The initial views demonstrated no abnormality in the parasternal long axis (PLAX) and apical four chamber views (A4C) as can be seen in Figure 2. Slight modification of the views, by angling the probe slightly posteriorly resulted in loss of the traditional A4C view but demonstrated loss of integrity of the interventricular septum (Figure 3). The rupture of the ventricular septum was visible towards the apical part of the septum as seen in the $\mathrm{A} 4 \mathrm{C}$ views and also more to the posterior aspect of the septum as visible on the parasternal short axis view (Figure 4). The use of colour doppler to interrogate the rupture also demonstrated the turbulent flow across the defect with a significant left to right shunt (Figure 4). The images also demonstrated no significant right ventricular dilatation and good right ventricular systolic function at the time. His left ventricular function remained good despite the insult, and he appeared to have an ejection fraction of $>40 \%$ estimated by eyeballing.

Ambulance had been held up for approximately $25 \mathrm{~min}$ to allow for the multi-disciplinary conversation regarding the optimum destination. He had invasive pressure monitoring arranged during this time along with a metaraminol infusion set-up for transfer.

The finding of the VSR complicated the referral principally due to unavailability of cardiothoracic surgical or extracorporeal membrane oxygenation (ECMO) services at the referral hospital. Following discourse between the EP the cardiothoracic surgeon and the cardiologists at the two hospitals, the verdict effectuated by the cardiologists was to proceed as initially planned because the cardiac catheterisation laboratory had already been activated with a STEMI protocol. They were advised that it would be prudent to have an anaesthetist on standby as clinical deterioration was almost a certainty.

At the laboratory during percutaneous coronary intervention (PCI), IABP was inserted and imaging revealed left anterior descending artery (LAD) occluded mid vessel and right coronary artery (RCA) occluded midvessel (Table 1). Attempts at PCI failed to achieve any increase in flow and patient had a pulseless electrical activity (PEA) cardiac arrest from which he had return of spontaneous circulation (ROSC) after 2 min of cardiopulmonary resuscitation (CPR). $\mathrm{He}$ had his airway secured and after another PEA arrest from which he regained ROSC after $5 \mathrm{~min}$ of $\mathrm{CPR}$, it was decided to transfer him intensive care unit (ICU) at the second referral hospital under the care of the cardiothoracic team.

He was operated on $12 \mathrm{~h}$ later (Table 2) with left internal mammary artery to left anterior descending artery graft (LIMA $\rightarrow$ LAD) and bovine pericardial patch repair via a left ventriculotomy. He developed significant right heart distension with akinesis, severe left ventricular dyskinesis with a persisting septal defect. Further attempts at securing the patch through a 


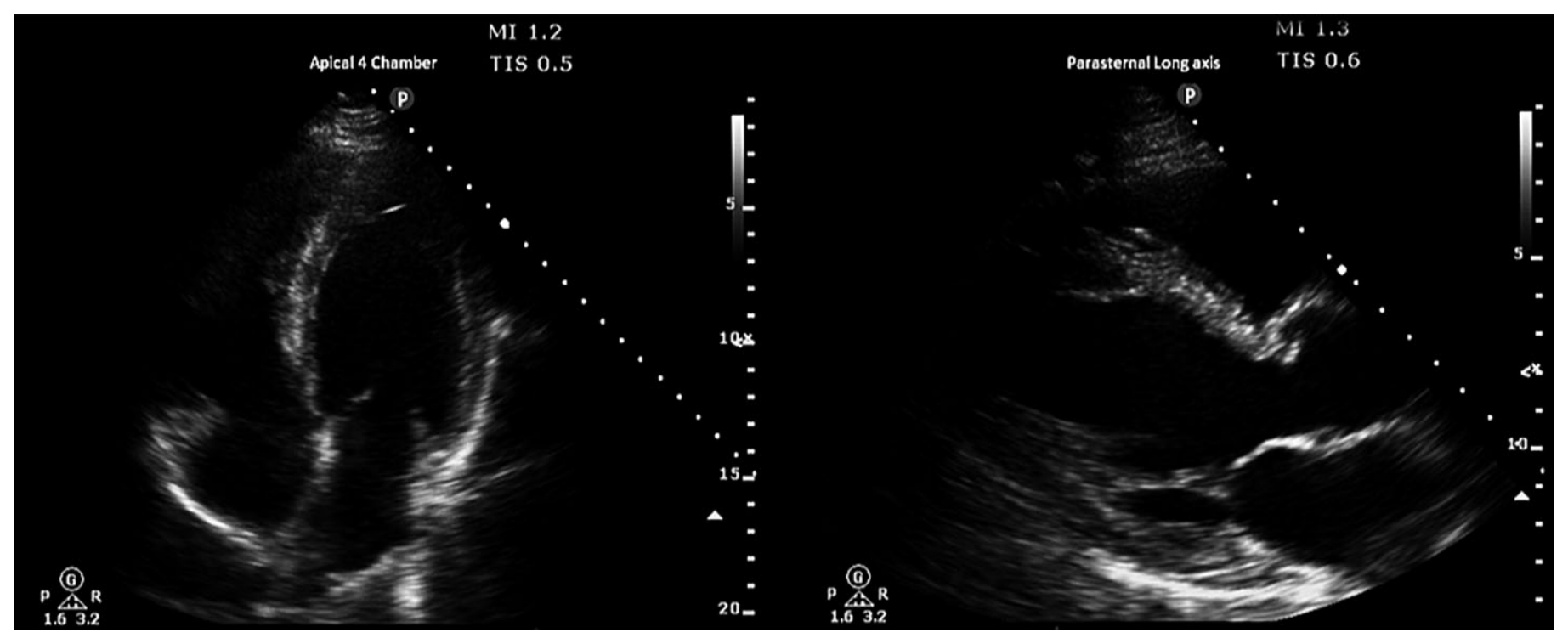

Figure 2: Parasternal Long Axis and A4C Views not Revealing the Ventricular Septal Rupture.

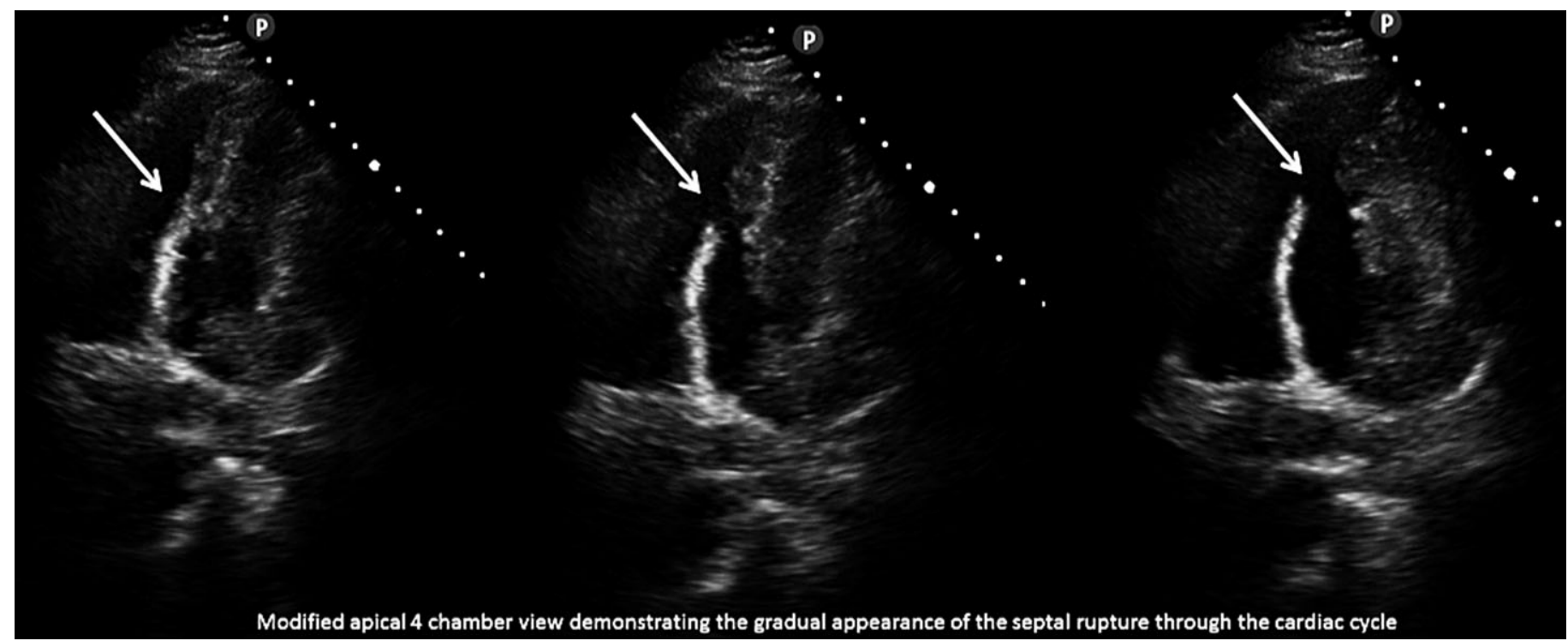

Figure 3: Three Images on a Modified A4C View that Shows the Ventricular Septal Rupture.

right atriotomy also failed and a consensus decision was taken for a one way wean off cardiopulmonary bypass and transfer to ICU for comfort care.

\section{Discussion}

Ventricular septal rupture was first recognised as a complication of AMI at autopsy by Latham ${ }^{3}$ in 1847 . The first surgical repair of VSR was described in 1957 by Cooley et al. ${ }^{4}$ The first long-term survivor was reported in 1963 by Payne et al. ${ }^{5}$ In a publication of the Society of Thoracic Surgeons National Database the early operative mortality was $54.1 \%$ (for repair within 7 days of $\mathrm{MI}$ ), which remains by large the highest of any other cardiac surgery. ${ }^{6}$ Despite the declining incidence, mortality from VSR remains high (41-80\%) and appears mostly unchanged worldwide in the last few decades.

Despite the high surgical mortality, the 2013 American College of Cardiology Foundation/American Heart Association guidelines recommend emergent surgical repair of post-MI VSR, regardless of hemodynamic status due to the risk of abrupt expansion of the rupture site and sudden hemodynamic collapse in a previously stable patient. ${ }^{7}$ The European Society of Cardiology state that early surgery should be performed in all patients with severe heart failure that does not respond rapidly to aggressive therapy, but delayed elective surgical repair may 


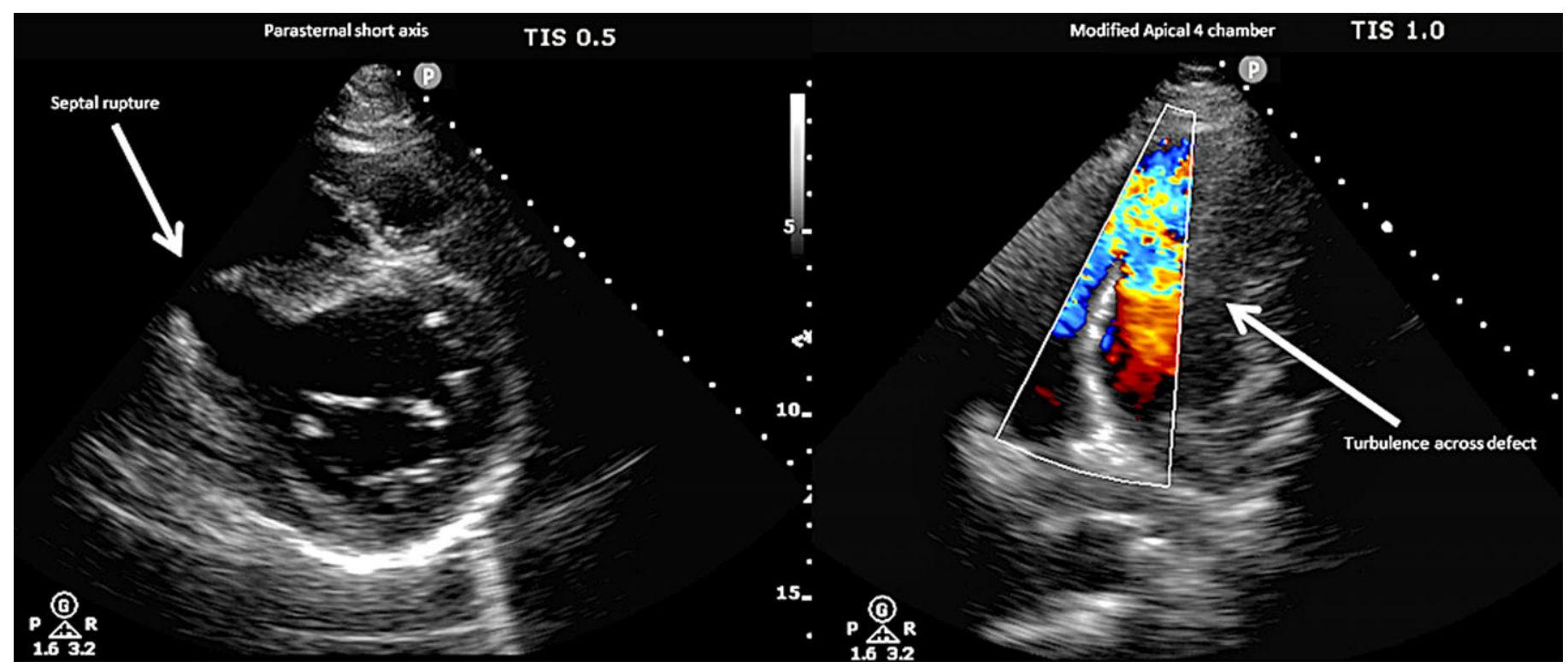

Figure 4: Parasternal Short Axis View Showing a Posterior Ventricular Septal Rupture and Doppler Shows the Left to Right Shunt Across the Ventricular Septal Rupture.

Table 1: Angiogram findings.

\begin{tabular}{|l|}
\hline LMCA: Patent \\
\hline LAD: Occluded in mid vessel \\
\hline LCx: Co-dominant. Mild disease \\
\hline RCA: Occluded in mid vessel \\
\hline LVgram: Large VSD. EDP $23 \mathrm{mmHg}$ \\
\hline
\end{tabular}

$L A D$, left anterior descending artery; LCx, left circumflex artery; LMCA, left main coronary artery; RCA, right coronary artery; VSD, ventricular septal defect.

Table 2: Intra-operative findings.

\begin{tabular}{|l|}
\hline Significant inferior wall infarction with necrosis \\
\hline Significant LV and RV dysfunction \\
\hline Significant coronary artery atherosclerosis \\
\hline Significant septal infarction with necrosis \\
\hline Large ventricular septal defect extending from Apex to Mitral Annulus \\
\hline
\end{tabular}

be considered in patients who respond well to aggressive heart failure therapy. ${ }^{8}$

Ours is perhaps the first reported case in Australia of acute post-MI VSR diagnosed through echocardiography performed by an EP. With cardiogenic shock and a low-output state, a palpable thrill and an audible murmur may be difficult to identify because the turbulent flow across the defect is reduced. Thus having echocardiography skills will prove critical in confirming the diagnosis, where clinical examination may be inconclusive.
Three types of VSR have been described: type I, being an abrupt tear in the ventricular wall without thinning, type II is defined as infarcted myocardium that erodes before rupture and is covered by thrombus and type III VSR describes the perforation of a previously formed aneurysm. The presented case is likely Type II with some thinning and scarring of the septum seen suggestive of infarcted myocardium. ${ }^{9}$

It must be appreciated that a VSR may not be visible in the standard echocardiographic imaging planes as has been demonstrated in Figure 2. It is imperative that the clinician sonographer manages to perform appropriate sweeps through the regions and obtain non-traditional windows to interrogate thoroughly for structural damage.

These cases would likely go on to develop decompensated heart failure as the evolution of this disease process. The left to right shunt would result in right ventricular overload, increased pulmonary blood flow and thus increased volume overload of the left atrium and left ventricle. As forward flow wanes, systemic vasoconstriction occurs increasing the left to right shunt and further impacting the left ventricular systolic function. Biventricular failure will be the ultimate terminal event.

Although pulmonary-to-systemic blood flow ratio (Qp/Qs) could be performed, it is generally not necessary as an acute VSR would be closed acutely. To adequately obtain this ratio, right ventricular outflow tract (RVOT) diameter, left ventricular outflow tract (LVOT) diameter, velocity-time integral (VTI) of the RVOT and VTI of the LVOT would need to be calculated. Poor windows in a critically ill patient are not always conducive to securing this data and capturing this information should in no way delay appropriate disposition of this patient. 


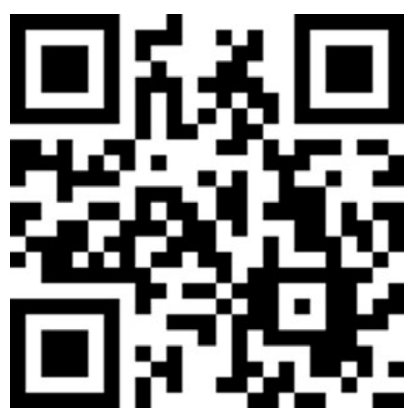

Figure 5: QR Code for Video of the Echocardiogram.

There is presently a dearth of high-quality evidence to direct clinicians to a single strategy in the management of such patients presenting with post-AMI VSR with decompensated cardiogenic shock. ${ }^{10}$ All approaches reported have extremely high mortality especially in acute post-AMI VSR cases of posterior location of a large VSR presenting within the first $24 \mathrm{~h}$ of AMI, as was observed in our specific case. ${ }^{11}$ Veno-arterial ECMO is being used categorically pre-operatively in such rare presentations to allow for restoration of hemodynamic stability as presented by McLaughlin et al. ${ }^{12}$ There is also an increased interest in the use of transcatheter device closure for VSR. At present it seems to be well suited for treatment of patch leaks after surgical repair. Thus evidence is forming towards ECMO and assist devices for early stabilization followed by early surgical repair with transcatheter closure of patch leaks as needed. ${ }^{13}$

The role ultrasound played in establishing this diagnosis so early in the patient journey illustrates the importance of having the skill to perform an echo in clinical medicine. Faced with a similar situation, I would possibly insist on transfer to a hospital with cardiothoracic services at the first instance.

\section{Key messages}

- Bedside point of care echocardiography is extremely useful in capturing the diagnosis of acute post-MI VSR, in patients who are invariably too unstable to have a formal echocardiography in the echo lab. Thus enabling the formulation of an optimal therapeutic strategy in cardiogenic shock. Basic echocardiography is a skill EP's must incorporate in their armamentarium as this is more likely to expedite the scan when compared to a formal echocardiography. This is exemplified here as the lack of clinical signs may not always raise the suspicion sufficiently and routine bedside echocardiography for MI's is thus not unwarranted.

- Immediate multi-disciplinary conference call should ideally be initiated with the EP, cardiologist, cardiothoracic surgeon, intensivist and possibly a cardiac anaesthetist. These individual case-tailored discussions are likely to provide the best opportunity to establish the therapeutic strategy progressing to definitive surgery.
- For centres with the adequate resources, ECMO or insertion of ventricular assist devices (VAD's) ought to be considered early and this might help with recovery of any multi organ failure and provide a better opportunity to create a better cardiac tissue environment for patch fixation.

\section{Competing interests}

The authors declare that they have no competing interests.

\section{Additional images \& video- QR code}

Figure 5

\section{Consent}

Written informed consent was obtained from the patient's wife for publication of this case report and accompanying images.

\section{References}

1 Novak M, Hlinomaz O, Groch L, Rezek M, Semenka J, Sikora J, et al. Ventricular septal rupture - a critical condition as a complication of acute myocardial infarction. J Crit Care Med 2015; 1(4): $162-6$.

2 Walts P, Gillinov A. Survival after simultaneous left ventricular free wall, papillary muscle, and ventricular septal rupture. Ann Thorac Surg 2004; 78(5): e77-8.

3 Latham PM. Lectures on subjects connected with clinical medicine, comprising diseases of the heart, vol. 2. London: Longman Rees; 1845. 168-74.

4 Cooley DA, Belmonte BA, Zeis LB, Schnur S. Surgical repair of ruptured interventricular septum following acute myocardial infarction. Surgery 1957; 41: 930.

5 Payne WS, Hunt JC, Kirklin JW. Surgical repair of ventricular septal defect due to myocardial infarction. Report of a case. JAMA 1963; 183: 603-5.

6 Arnaoutakis G, Zhao Y, George T, Sciortino C, McCarthy P, Conte J. Surgical repair of ventricular septal defect after myocardial infarction: outcomes from the society of thoracic surgeons national database. Ann Thorac Surg 2012; 94(2): 436-44.

7 O'Gara PT, Kushner FG, Ascheim DD, Casey DE Jr, Chung MK, de Lemos JA, et al. 2013 ACCF/AHA guideline for the management of ST-elevation myocardial infarction: a report of the American College of Cardiology Foundation/American Heart Association Task Force on Practice Guidelines. Circulation 2013; 127(4): e362-425.

8 Esc S. The task force for the management of acute myocardial infarction in patients presenting with ST-segment elevation of the European Society of Cardiology (ESC) 2017 ESC guidelines for the management of acute myocardial infarction in patients presenting with ST-segment elevation. Russ J Cardiol 2018; 5: 10358.

9 Bansal RC, Eng AK, Shakudo M. The role of echocardiography in the assessment of ventricular septal rupture post myocardial infarction. J Diagn Med Sonogr 2017; 33(4): 291-4.

10 Singh V, Rodriguez A, Bhatt P, Alfonso C, Sakhuja R, Palacios I, et al. Ventricular septal defect complicating ST-elevation myocardial infarctions: a call for action. Am J Med 2017; 130(7): 863.e112 . 
11 Asai T. Postinfarction ventricular septal rupture: can we improve clinical outcome of surgical repair? Gen Thorac Cardiovasc Surg 2016; 64(3): 121-30.

12 McLaughlin A, McGiffin D, Winearls J, Tesar P, Cole C, Vallely M, et al. Veno-arterial ECMO in the setting of post-infarct ventricular septal defect: a bridge to surgical repair. Heart Lung Circ 2016; 25(11): 1063-6.
13 Wilson WM, Horlick EM. Management of post-myocardial infarction ventricular septal rupture. EuroIntervention 2016; 12(Suppl $\mathrm{X)}$ : X18-23. 